\title{
REVISTA
}

Revista Educación

ISSN: 0379-7082

ISSN: 2215-2644

revedu@gmail.com

Universidad de Costa Rica

Costa Rica

\section{Fondos de conocimiento e identidad: Una vía sociocultural de continuidad educativa}

\author{
Brito Rivera, Luis Fernando; Subero Tomás, David; Esteban-Guitart, Moisés \\ Fondos de conocimiento e identidad: Una vía sociocultural de continuidad educativa \\ Revista Educación, vol. 42, núm. 1, 2018 \\ Universidad de Costa Rica, Costa Rica \\ Disponible en: http://www.redalyc.org/articulo.oa?id=44051918005 \\ DOI: http://dx.doi.org/10.15517/revedu.v42i1.23470
}

Esta obra está bajo una Licencia Creative Commons Atribución-NoComercial-SinDerivar 3.0 Internacional. 


\title{
Fondos de conocimiento e identidad: Una vía sociocultural de continuidad educativa
}

\section{Funds of Knowledge and Identity: A Sociocultural Approach of Educational Continuity}

Luis Fernando Brito Rivera [1]

Escuela Normal de Texcoco, México

DOI: http://dx.doi.org/10.15517/revedu.v42i1.23470

luisfernandobrito@yahoo.com.mx

Redalyc: http://www.redalyc.org/articulo.oa?id=44051918005

David Subero Tomás [2]
Universidad de Girona, España
david.subero@udg.edu

Recepción: 10 Marzo 2016

Aprobación: 23 Marzo 2017

\author{
Moisés Esteban-Guitart [3] \\ Universidad de Girona, España \\ moises.esteban@udg.edu
}

\section{RESUMEN:}

El impacto de la globalización y la llamada sociedad de la información ponen en cuestión los parámetros tradicionales sobre los cuales hemos definido el aprendizaje: dónde se produce, con quién, cómo y para qué. Uno de los ejes sobre los que distintos estudios parecen estar de acuerdo es el carácter situado y distribuido que tienen los aprendizajes, y con ello la necesidad de establecer continuidades educativas entre las distintas experiencias de aprendizaje tanto dentro como fuera de la escuela. En este contexto, proponemos una relación teórico-metodológica entre los fondos de conocimiento e identidad y la historia oral para: a) producir una continuidad, en el proceso educativo, a través de elementos socioculturales de alto significado para el alumnado de educación básica; b) replantear la enseñanza como una actividad fundamentada en la interactividad sociocultural desde la misma formación inicial del profesorado. El trabajo deriva del primer ciclo de una investigación basada en el diseño desarrollada en una escuela normal en México con profesorado en formación. Las conclusiones preliminares apuntan a la posibilidad de establecer un proceso de formación inicial centrado en el desarrollo de continuidades educativas durante las experiencias de acercamiento a la práctica docente. Este ejercicio ha ayudado a ajustar la estrategia de investigación sobre lo que se ha denominado didáctica cultural inclusiva. Palabras Clave: Fondos de conocimiento e identidad, historia oral, nueva ecología del aprendizaje, continuidad educativa, formación del profesorado.

\section{ABSTRACT:}

The impact of globalization and the so-called information society put in question the traditional parameters in which we have defined learning: where it occurs, with whom, how and for what reason. One of the axis on which different authors seem to agree is the character and distributed that have the programming, and with it the need to establish educational continuities between the various experiences of learning both inside and outside of the school. In this context, we propose a theoretical-methodological relation between the funds of knowledge and identity and oral history for: a) to produce a continuity in the educational process, through socio-cultural elements of high significance for the students of basic education; b) reconsider the teaching as an activity based on the sociocultural interactivity from the same initial teacher training. The work is a derivative of the first cycle of an investigation based on the design developed in a normal school in Mexico with teachers in training. Preliminary conclusions point out the possibility of establishing a process of initial training focused on the development of educational continuities during the experiences of approach to the teaching practice. This exercise has helped to adjust the strategy for research on what has been called inclusive cultural didactics.

KEYWORDS: funds of knowledge and identity, oral history, new ecology of learning, educational continuity, teacher training.

\section{Notas DE AUTOR}

[1] Doctor por la Universidad Autónoma Chapingo, México. Doctorando por la Universidad de Girona, España (Doctorado Interinstitucional en Psicología de la Educación)

[2] Profesor asociado. Departamento de Psicología. Instituto de Recerca Educativa. Facultad de Educación y Psicología.

[3] Profesor agregado. Departamento de Psicología. Instituto de Recerca Educativa. Facultad de Educación y Psicología. 


\section{INTRODUCCIÓN}

Este artículo ${ }^{[4]}$ es un ejercicio de reflexión basado en cuatro ejes. El primero consiste en un análisis sobre las dinámicas de cambio en el estudio y la definición del aprendizaje en el contexto de la "nueva ecología del aprendizaje" (Coll, 2013a). El interés es señalar algunas circunstancias de cambio en la forma de aprender, ya que los contextos de educación no formal cobran mayor significación y requieren de nuevas formas de conectarlos a las actividades educativas formales. Lo anterior supone recuperar el concepto mesosistema (Bronfenbrenner, 1987), según el cual el potencial evolutivo de un contexto o escenario de aprendizaje aumenta en la medida en que sea capaz de generar continuidades educativas con otros escenarios educativos, en los que las personas participan activamente. Este fenómeno modifica, de manera sustancial, la identidad de docentes y estudiantes, así como las funciones, roles, significados y sentidos de la actividad de enseñar y aprender.

El segundo eje apuntó a resolver cuál sería la manera de generar continuidades educativas desde la misma formación inicial del profesorado con el objetivo de incidir en los procesos de aprendizaje de sus estudiantes de educación básica. Parte de la respuesta se ubicó en el marco teórico-metodológico de los "fondos de conocimiento e identidad" para señalar las continuidades y discontinuidades en la relación profesoradoalumnado, especialmente de aquel estudiantado sub-representado por diversas razones (escasos ingresos, minoría étnica, clase social baja, etc.). El interés fue poner en marcha un proceso de formación docente congruente con las necesidades de aprendizaje del alumnado, así como de sus conocimientos, habilidades y destrezas emanadas de sus recursos y prácticas culturales. De tal modo que se rescatara la historia de vida del alumnado para la conformación del pensamiento a través de la mediación de las actividades de práctica y diálogo (Moll, 2014).

El tercer eje, esta vez desde la perspectiva didáctica, fue cómo conectar los diversos contextos de vida de estudiantes de secundaria para que el personal docente en formación propicie aprendizajes significativos. Fue así como se recurrió a la historia oral, pues en sus fundamentos existe cierta colindancia con los fondos de conocimiento e identidad. Es decir, en ambas perspectivas se reconocen las experiencias de vida de las personas como fuentes válidas de conocimiento. A esta interrelación se le denominó "didáctica cultural inclusiva" (DCI), ya que cada estudiante, considerando su diversidad cultural, ha de ser reconocido como un sujeto activo dentro de las prácticas y pedagogías escolares. Perspectiva que requiere que el profesorado se convierta en aprendiz de sus estudiantes, así como de un cambio profundo en las relaciones de enseñanzaaprendizaje puesto que, tanto alumnado como profesorado son actores críticos de su propia actividad educativa.

El cuarto eje se basó en identificar las posibles continuidades/discontinuidades educativas sobre los sentidos y significados que los grupos docentes en formación le dan a: a) la relación profesorado-alumnado; b) relación escuela-familia y c) la concepción que se tiene sobre el alumnado. Este ejercicio es parte del primer ciclo de una investigación basada en el diseño que tiene como propósito desarrollar innovaciones en el diseño de secuencias didácticas que interconecten aprendizajes entre contextos educativos formales e informales. Las dinámicas generadas, mediante grupos focales, permitieron tener un acercamiento significativo con los grupos docentes en formación. Este ejercicio ayudó a establecer los propósitos del segundo ciclo de la investigación que, en este caso, fue la preparación de una inducción teórico-metodológica sobre la perspectiva de los fondos de conocimiento e identidad, así como de la historia oral.

Este proceso allana el camino para el diseño de secuencias didácticas interconectadas a la ecología del aprendizaje de estudiantes de secundaria, propósito del tercer ciclo de la investigación. Los resultados de los grupos focales sugieren: a) que el profesorado en formación, a pesar de su incipiente trayecto formativo, tiene una concepción bastante tradicional sobre la relación docente-estudiante; b) la relación entre la escuela y la familia es difusa y poco significativa; c) el alumnado de educación básica es definido como un sujeto pasivo que ha de esperar instrucciones para aprender. No obstante, el profesorado en formación también manifestó 
que es necesario constituir vías de renovación, tanto en la relación directa con el alumnado de educación básica, como en la trayectoria de la formación inicial. En otras palabras, se identificó una discontinuidad educativa en la formación inicial del profesorado que puede ser el marco de un proceso de innovación para la formación docente.

\section{EL CONTEXTO DE CAMBIO EN LA EDUCACIÓN}

Mucho se ha debatido sobre ciertos cambios en los contextos social, económico, político y cultural, debido, en parte, a la presencia y uso de las tecnologías de la información y comunicación (TIC), la formación de la economía global, así como de procesos de cambio cultural (Castells, 1994; Tedesco, 2011). Nos encontramos en un momento donde las TIC y la presencia de internet amplían las perspectivas del espacio público del conocimiento (Organización de las Naciones Unidas para la Educación, la Ciencia y la Cultura, 2005), lo mismo que los flujos de información y las posibilidades de conectividad, entre los distintos propósitos de orden personal, social, económico y de trabajo, van reorganizando las esferas de la actividad humana (Organisation for Economic Cooperation and Development, 2012). Los puntos anteriores implican reconocer que los conocimientos y saberes son cambiantes y dinámicos, ya que ninguno de estos es definitivo en el sentido tradicional, puesto que se encuentran distribuidos y situados en el sistema de la actividad humana (Engeström, 1987; Engeström, 1999; Leontiev, 1984; Radzikhoskii, 1997).

\section{Escuela, educación y aprendizaje: Perspectivas de interconexión y retos de cambio}

Ante este escenario, la escuela ya no es el único contexto ni el más importante para aprender. Ello se debe, en parte, a que los sujetos pueden aprender casi en cualquier momento y espacio, mediante el uso de las TIC dadas sus características de movilidad y ubicuidad, así como por la utilización simultánea y convergente de diversos lenguajes (Coll, 2013b). Situación que implica reconocer nuevos contextos educativos y, por ende, una nueva ecología del aprendizaje (Barron, 2010; Coll, 2013a).

Desde esta perspectiva, la escuela y la educación tienen nuevos debates sobre cómo crear posibilidades de acceso a las ideas, conversaciones, herramientas y redes sociales de los contextos informales de aprendizaje con el objetivo de enriquecer el desarrollo de las personas. Se trata de un fenómeno complejo que implica un replanteamiento importante sobre las funciones de la escuela: el reto es la búsqueda de oportunidades de aprendizaje para que el alumnado fortalezca sus intereses y satisfaga sus necesidades individuales contando con la responsabilidad compartida del centro escolar y del resto de los agentes sociales (Coll, 2010).

En este escenario consideramos que las perspectivas generales de las instituciones de educación formal se fundamentan en: a) la importancia del aprendizaje a lo largo y ancho de la vida; b) la tendencia a la informalización de las oportunidades de aprendizaje; c) la importancia de las trayectorias personales de aprendizaje como vía de acceso al conocimiento; d) la adquisición de competencias genéricas y transversales relacionadas con la capacidad para aprender, así como la tendencia creciente de los enfoques curriculares basados en competencias -ECBC- (Coll, 2009b, 2010); e) la tendencia creciente a la personalización del aprendizaje (Coll, 2013a); f) la reubicación de la escuela en la red de contextos de aprendizaje por los que transita el alumnado (Coll, 2013b), y g) el efecto de las TIC en la aparición de nuevos entornos educativos (Bustos y Coll, 2010).

Por ello, es importante la reubicación y redefinición de la escuela dentro de la red de contextos de aprendizaje por la que transitan las personas, y en específico, sobre cómo generar un proceso educativo mediado por elementos socioculturales de alto significado y que, con el replanteamiento de la enseñanza desde la misma formación inicial del profesorado, pueda producir continuidades entre las prácticas y recursos culturales de los diferentes contextos de vida del alumnado. 
Docentes y estudiantes: hacia el desarrollo de aprendices competentes

Como ya se mencionó, la dinámica de interconexión genera cambios en las prácticas y actividades educativas, transformando las necesidades por aprender, así como los propósitos y objetivos de la acción formadora. Esto implica que el alumnado sea más activo, autónomo y estratégico para generar, determinar y redistribuir su propio itinerario de aprendizaje a través de la interconexión con sus pares y con los distintos actores y contextos de aprendizaje. Por su parte, la función del profesorado sería la de prestar soporte y guía en la construcción de conocimientos y significados de aprendizaje, a través del desarrollo y proporción tanto de ambientes formativos enriquecidos como de ayuda educativa ajustada. Situación que implica cambios, tanto en la enseñanza como en los procesos de formación inicial docente. En lo que toca a la escuela, esta tendría que ser redefinida en su función y organización, lo que supone adoptar modelos de gestión y administración educativa acordes a estas nuevas condiciones. Siendo así, el interés sería potenciar el aprendizaje por medio de procesos formativos flexibles y dinámicos para alinear, tanto los motivos de las instituciones educativas como los del alumnado (Coll, 2009a, 2013b). La incorporación, en el proceso educativo formal, de los mundos de vida de estudiantes, considerando sus prácticas y recursos culturales, nos parece una iniciativa que refuerza el mesosistema y está en consonancia con las características de la nueva ecología del aprendizaje.

En este sentido, parte del proyecto que aquí se presenta tuvo el objetivo de establecer una continuidad entre las prácticas y recursos culturales del alumnado de secundaria y las secuencias y planeaciones didácticas que docentes en formación elaboraron a partir de las necesidades de aprendizaje identificadas en sus contextos de práctica. Todo esto con un doble propósito: generar una continuidad entre el proceso educativo formal y la cultura del alumnado de secundaria, así como replantear la enseñanza como una actividad fundamentada en la interactividad sociocultural desde la misma formación inicial. Es así como hemos recurrido a los "fondos de conocimiento e identidad" y a la "historia oral" como vía estratégica para incidir y conectar la ecología del aprendizaje tanto de docentes en formación como de sus estudiantes.

\section{LOS FONDOS DE CONOCIMIENTO E IDENTIDAD}

El concepto de "fondos de conocimiento" tiene su origen en la antropología y parte de los estudios llevados a cabo en los años ochenta por Carlos Vélez-Ibañez y James Greenberg (1992) en la Universidad de Arizona, con énfasis en los intercambios económicos y relacionales de los grupos inmigrantes mexicanos de clase obrera en los Estados Unidos de América, especialmente en la ciudad de Tucson, Arizona. Con estos antecedentes se conforma, a inicios de los años noventa, el proyecto educativo "fondos de conocimiento" contando con los trabajos del psicólogo educativo Luis C. Moll así como de la antropóloga educativa Norma González. El proyecto parte de un marco sociocultural, y pone énfasis en cómo las prácticas culturales y los recursos que las median generan y desarrollan conocimiento. El concepto antropológico originario basado en intercambios económicos, trabajos y relaciones sociales se fue reconfigurando con el objetivo de recoger e identificar conocimientos, habilidades y destrezas a partir de los recursos culturales existentes tanto en las familias como en la comunidad (González, 1995). Por tal, los fondos de conocimiento se han definido como "cuerpos de conocimiento y habilidades culturalmente desarrollados e históricamente acumulados esenciales para los hogares, el desarrollo individual y el bienestar” (Moll, Amanti, Neff y González, 1992, p.133).

El programa "fondos de conocimiento" se plantea como una estrategia fundamental para incidir en la búsqueda de las prácticas culturales de dicho alumnado reconociéndolas e incorporándolas como aprendizajes válidos dentro de la escuela. Desde esta perspectiva, la problemática sobre el fracaso escolar ya no se focaliza en las capacidades del alumnado ni en sus familias sino en el desajuste de la gestión de la comunicación entre el alumnado, la familia y la escuela. Esta perspectiva teórica obliga a modificar la organización de la actividad docente y de la institución escolar acercándola a la experiencia cultural del alumnado. 
Recientemente, algunos avances teóricos y metodológicos han sido replanteados en relación con los fondos de conocimiento. Una de estas posturas ha sido propuesta por Esteban-Guitart (2012a, 2014) y consiste en tomar en cuenta los fondos de identidad del alumnado, como complemento de los fondos de conocimiento, poniendo énfasis en la construcción de la identidad a través de factores culturales, actividades y prácticas significativas.

Los fondos de identidad son artefactos, tecnologías o recursos, históricamente acumulados, culturalmente desarrollados y socialmente distribuidos y transmitidos, esenciales para la autodefinición, autoexpresión y autocomprensión de las personas (Esteban-Guitart, 2012a). Para el autor, el reto consiste en partir de estos fondos de identidad, que expresan los conocimientos previos, intereses y motivaciones del aprendiz, para que el personal docente pueda detectarlos, reconocerlos e incorporarlos como prácticas pedagógicas y recursos legítimos dentro de las instituciones educativas. En otras palabras, se espera aumentar la motivación, implicación y rendimiento escolar a partir del uso de los conocimientos, destrezas e intereses de los alumnos y alumnas que expresan fondos particulares de identidad. Estos fondos, al ser incluidos en las actividades escolares, pueden ser una ruta de continuidad educativa entre los diferentes contextos de aprendizaje del alumnado.

El individuo, al darle sentido y continuidad a sus experiencias vitales y de aprendizaje, va construyendo su identidad. Esta, al ser entendida como un proceso cultural, mediado por las interacciones con la sociedad, podrá ser reconstruida a través de narraciones, historias y relatos de vida para cohesionar y darle un propósito a la experiencia de vida pasada, presente y futura (Bruner, 2003, citado por Esteban-Guitart, 2012b). Es así como proponemos fundamentar una metodología basada en historias orales con el propósito de interconectar los fondos de conocimiento e identidad del alumnado de secundaria, por medio de las actividades didácticas de docentes en formación. En otras palabras, a través del desarrollo de la historia oral se podrán recoger las prácticas culturales y hacer emerger los recursos que las median. Es decir, un dialogo entre docentes en formación y estudiantes de secundaria como recurso para reconocer sus identidades (tanto del profesorado como del alumnado) y consolidar una interactividad educativa ajustada a las condiciones socioculturales de la ecología del aprendizaje del contexto escolar y comunitario.

Es más, la identificación de los fondos de conocimiento e identidad del alumnado de secundaria, a través de la construcción de su historia oral, permitirá conectar los recursos didácticos a las necesidades y sentidos más auténticos del sujeto aprendiz. Este efecto flexibilizará y personalizará las prácticas educativas, incluyendo la interculturalidad de todos los sujetos implicados. De esta manera, se espera que el proceso de la formación inicial se transforme cualitativamente, pasando de la reproducción de ciertas prácticas a la constitución de una identidad del profesorado que aprende mediante el reconocimiento de la identidad de sus estudiantes de educación básica. A esto lo hemos definido como aprender a enseñar a aprender; fundamento significativo de lo que hemos denominado didáctica cultural inclusiva.

\section{La historia oral: La vía etnográfica de los fondos de conocimiento e identidad}

La historia oral (HO) es una metodología para crear fuentes sobre cómo los individuos (actores, sujetos, protagonistas, observadores) se perciben o son afectados por los diferentes procesos históricos de su tiempo (Collado, 2006). El análisis producido por la $\mathrm{HO}$ fortalece el conocimiento de lo social, ya que, por medio del discurso biográfico, su autor o autora estructura, desestructura y reestructura la realidad que percibe. Así, la $\mathrm{HO}$ es adecuada para abordar problemáticas donde la experiencia humana, es accesible solo por medio de la fuente oral, ya que por su naturaleza no queda registrada en las fuentes escritas. Tal es el caso de los fondos de conocimiento e identidad del alumnado de secundaria.

La utilización de la historia oral busca valorar y reconocer, en el aula y en las prácticas culturales al alumnado, así como a sus familias y comunidades, ya que tienen una historia que contar, pues sus vidas también son conocimiento. La historia oral reivindica una visión crítica sobre el presente, así como un sentido 
prospectivo sobre el futuro que deseamos vivir. No se puede pensar lo global, en menoscabo de lo regional y local, cuando son excluidos los conocimientos e historias de diversos agentes sociales como la persona migrante, la madre soltera, el obrero, el ama de casa o la misma juventud que en sus prácticas culturales buscan la definición de su identidad. Sujetos que, "de una u otra manera, han sido producidos por las condiciones y dinámicas desiguales de una sociedad en constante transformación, evolución y cambio" ( Brito, 2014, p. 13).

El objetivo es reconstruir las prácticas sociales destacando aspectos políticos, económicos, familiares, laborales, psicológicos, así como hábitos, actividades, percepciones y costumbres de la vida cotidiana. Lo que interesa es saber cómo estudiantes y sus familias desarrollan las redes sociales que les conectan con su ambiente y cómo esta situación produce el intercambio de recursos como los fondos de conocimiento e identidad. A partir del uso de la historia oral, los cuerpos docentes en formación son capaces de interactuar haciendo emerger y comprendiendo los recursos, históricamente acumulados, culturalmente desarrollados y socialmente distribuidos que definen a sus estudiantes.

El vínculo entre los fondos de conocimiento e identidad y la historia oral queda establecido en la medida en que se logre producir una continuidad entre el contexto educativo formal con aquellos aspectos socioculturales que el alumnado comparte con otros nichos de aprendizaje no formal. En este sentido, la continuidad se establece al momento en que el profesorado reconstruye la historia de vida sus estudiantes y logra aprender cuáles son sus aspectos más significativos. Esto haría posible que las actividades de enseñanzaaprendizaje logren una continuidad y, por ende, mejores niveles de progreso académico en el alumnado, así como desarrollar una actitud más reflexiva en el profesorado sobre sus propias prácticas. Visto así, tanto estudiante como docente podrán desarrollarse como actores críticos de su propia actividad educativa.

\section{CONTINUIDADES Y DISCONTINUIDADES EDUCATIVAS: UNA EXPERIENCIA EN LA FORMACIÓN INICIAL DEL PROFESORADO}

En esta parte del texto nos interesa hacer una reflexión sobre las continuidades/discontinuidades que experimenta el profesorado en formación con respecto a los fondos de conocimiento e identidad del alumnado de secundaria durante el trayecto de las prácticas docentes. Los resultados expuestos en este apartado son parte del primer ciclo de una investigación basada en el diseño con el objetivo de fundamentar la didáctica cultural inclusiva como una actividad educativa incluyente, basada en las características culturales del alumnado de secundaria.

\section{Un ejercicio de innovación educativa fundamentado en la investigación basada en el diseño}

Durante el periodo de diciembre de 2014 a mayo de 2015, y en el marco de una estancia de intercambio de experiencias académicas y de investigación educativa entre la Escuela Normal de Texcoco, Estado de México y la Universidad de Girona, España, se desarrolló un proyecto de investigación e innovación educativa con el objetivo de fortalecer el proceso de formación inicial del profesorado. El propósito fue consolidar una secuencia didáctica ${ }^{[5]}$ que, fundamentada en los fondos de conocimiento e identidad del alumnado de secundaria, pudiera generar aprendizajes tanto en estudiantes como en docentes. Es decir, generar continuidades tanto en la interacción entre los actores educativos como entre sus contextos de referencia.

Fue así como se recurrió a la investigación basada en el diseño (DBR) con la idea de desarrollar una investigación capaz de incrementar nuestra comprensión acerca del fenómeno, fortaleciendo el desarrollo de aplicaciones útiles para la formación inicial del profesorado. Se trata de un estudio de base sociocultural para intervenir en situaciones de enseñanza comprendiendo el contexto naturalístico del fenómeno y generar, paralelamente, ciertos datos empíricos (Anderson y Shattuck, 2012; Barab y Squire, 2004). El interés fue 
fundamentar la noción de didáctica cultural inclusiva (DCI) como una estrategia de interacción e influencia educativa basada en los fondos de conocimiento e identidad del alumnado.

La DBR nos permitió diseñar un proceso de intervención lo suficientemente robusto para interpretar y contextualizar las prácticas docentes del profesorado en formación con el objetivo de enriquecer y refinar, tanto la teoría de los fondos de conocimiento e identidad como la noción de didáctica cultural inclusiva. Lo que nos ayudó a diseñar, posteriormente, un proceso de innovación más ajustado a los requerimientos y características de la práctica docente.

Otro apoyo de la DBR fue su capacidad de generar ciclos continuos de recolección y análisis de información en consideración de los factores contextuales que favorecen o inhiben la efectividad de la intervención (Garello, Rinaudo y Donolo, 2011). La investigación se planteó como un estudio piloto[6] desarrollado de manera colaborativa por todos los actores implicados, tanto en las decisiones sobre los objetivos como en la aplicación de técnicas e instrumentos, incluyendo resultados, propuestas y acciones a seguir (Alcocer, 1998).

El planteamiento general del proyecto implicó responder cómo producir una continuidad entre el proceso de formación inicial docente y los aprendizajes del alumnado de secundaria. El objetivo general fue fortalecer tanto las competencias de enseñanza del profesorado en formación como las competencias de aprendizaje del alumnado de secundaria. La primera fase del proyecto, que es la que se presenta en este artículo, consistió en que docentes en formación comprendieran el contexto cultural de sus estudiantes para identificar y comprender las posibles continuidades/discontinuidades en el proceso de enseñanza-aprendizaje.

En el diseño de intervención se integraron tanto las nociones de los fondos de conocimiento e identidad como la perspectiva de la historia oral. Se consideraron las características del contexto en las que se lleva a cabo las prácticas docentes de estos 16 profesores y profesoras en formación. Las técnicas de recolección de datos consistieron en redes semánticas naturales, grupos focales, autoinformes, cuestionarios abiertos, tareas y observaciones. En el siguiente apartado se presenta el análisis de los grupos focales del primer ciclo del proyecto.

\section{Las continuidades/discontinuidades educativas entre la formación y práctica docente}

Cada sesión se desarrolló por medio de la técnica de grupos focales. La intención fue que todo el grupo de docentes en formación participara de manera activa en la búsqueda de sentidos sobre aquello que se preguntaba. La dinámica de diálogo, en los grupos focales, fue empática y registrada en audio. La identidad de los sujetos participantes ha sido resguardada bajo el principio del anonimato por lo que, al momento de presentar algunas de las respuestas, se usan seudónimos.

La primera sesión buscaba comprender qué, dónde y con quién aprendía el alumnado de secundaria. El propósito fue comprender cómo se definía el papel del alumnado, el profesorado y la escuela. La segunda sesión consistió en indagar sobre los contextos de vida y aprendizaje por los que transitaba el alumnado, tales como la familia y la comunidad y si estos eran recursos válidos de aprendizaje. Finalmente, en la tercera sesión se les preguntó sobre la secuencia didáctica, específicamente sobre sus componentes y las actividades de aprendizaje que la conformaban. Nuestro interés fue conocer hasta qué punto las secuencias didácticas estaban conectadas con las experiencias de vida e intereses del alumnado.

En este trabajo se presentan los datos referentes a la sesión uno y dos, ya que el primer ciclo de la investigación aún no ha terminado. Desde la lógica de la DBR es necesario tener datos que nos ayuden a redefinir los objetivos de todo el conjunto de la investigación, así como rediseñar las fases de intervención. Los datos presentados son una selección de las transcripciones textuales de los grupos focales[7]. Se hizo una depuración con el propósito de seleccionar las opiniones más significativas del profesorado en formación participante, agrupándolas en tres categorías: a) definición de alumnado; b) relación entre profesoradoalumnado; c) relación escuela-familia.

Sobre la definición del alumnado: 
Luis Fernando Brito Rivera, et al. Fondos de conocimiento e identidad: Una vía sociocultural de co...

Frida:

El alumno aprende todos los días y en todos los lugares; pero es importante la guía de alguien para que esos aprendizajes no se tornen negativos.

Miriam:

Qué son capaces de hacer los alumnos...todo y nada. Todo, ... sólo si tienen la necesidad de aprender algo. Si no tienen la necesidad no hacen nada. Es tan triste la situación ... son como esponjas que esperan el conocimiento del maestro. Y eso es limitante ... porque no crea autonomía.

Moisés:

El alumno ha de cumplir con ciertas reglas y normas. El alumno ha de comportarse porque algo le están enseñando en la escuela. El alumno ha de responder a la sociedad porque por algo va a la escuela. Yo creo que en las escuelas los alumnos se sienten más prisioneros de la escuela que en otros lugares. Ellos han de cumplir con un reglamento (uniforme, corte de cabello, una manera de hacer). Ellos han de ser como la presentación de la escuela, y esa presentación en ellos significa no ser yo mismo.

Sole:

El alumno está determinado como sujeto dentro del sistema educativo ... pero el alumno es persona e individuo y tiene su propia interpretación del contexto. El alumno aprende cuando está en interacción con ese contexto y con quien lo rodea. Lo más importante para mi en el ... aprendizaje es el para qué; qué hago con ese aprendizaje.

Sobre la relación profesorado-alumnado:

Daniel:

Qué sería un alumno si solo lo dejamos con lo que aprende de sus compañeros o lo que aprende de sus padres. Sería una educación mediocre. No tendriamos personas con un aprendizaje cientifico... ¿para qué estamos aqui (los docentes)? ¿Para qué son las escuelas? Yo creo que las escuelas tienen el objetivo de formar personas con un conocimiento cientifico.

José:

Conocer a tu alumno, a quién tienes en frente porque son personas, tienen gustos y tienen intereses y tú tienes que buscar la manera de crear la confianza (hablar sobre los videojuegos y decirle que puede relacionarlo con las matemáticas) es importante decirselo no de manera formal o técnica sino con un vocabulario informal para que ellos digan: pues si que tiene razón. Entonces si que es importante esto porque me está ayudando. ... Y también fomentar que ellos puedan desarrollar aspiraciones (como ser el programador de algún videojuego) y para ello necesitan desarrollar ciertos conocimientos que se dan en la escuela.

Elena:

Estoy de acuerdo en que el alumno aprende en base a sus necesidades e intereses. El maestro selecciona la información [para] el alumno. Pero el alumno, de esa información, va a seleccionar lo que él quiere para simismo. La selección de información puede ser tanto académica como para su vida. Por ello todo depende del alumno y la información que requiere para su vida. La figura del maestro es más como una guía sobre la información que le da al adolescente.

David:

Yo entiendo que un alumno es una personita que aprende y enseña a la vez.... va a interactuar con el maestro, le va a enseñar algo nuevo al maestro. Yo vi en las prácticas cómo los alumnos enseñaron a la maestra a conectar una simple conexión de sonido ... el alumno le enseñó cómo hacer cosas al maestro".

Delfina:

El maestro ha de ser el mediador del conocimiento, porque si no lo es pues... ¿Qué estamos haciendo aqui?

Sobre la relación escuela-familia:

Luis:

El problema es que se le cede mucho la responsabilidad a la escuela como el único lugar donde tienen que aprender, pero el lugar donde más conocimientos generan es en casa. Son los fundamentos donde ellos pueden 
insertarse a la educación. Porque si tu no le enseñas a un niño como debe de aprender, como ha de comportarse... Si el alumno aprende por el solo pues ¿Qué hacemos aqui... estudiando cómo maestros?

Iliana:

"Creo que en todo momento se aprende, pero hay cosas que aprendemos y después las dejamos de practicar. Es un aprendizaje inútil y lo tiramos a la basura. Si se aprende algo en la escuela es importante que se retomen en las familias... Y que también en la escuela es importante que se retomen los valores que debe tener una persona integra.

Edmundo:

Pero la escuela no es nada más la responsable, tiene parte de responsabilidad junto a la familia y la sociedad.... En las prácticas veo que los padres van a la escuela porque están castigados y muy pocos para saber cómo están sus hijos. Pero, de esos hijos que veo que sus padres están interesados es porque hay algo que los impulsa a los muchachos para que salgan; yo estoy viendo por mi familia y por mi porvenir. Se les está dejando la responsabilidad a otras personas y nadie quiere asumir la responsabilidad.

Regla:

Les preguntan los padres por las notas y no por los aprendizajes. Creo que ello no tiene mucho sentido. En cuento a los aprendizajes ellos (los alumnos) no son conscientes de que están aprendiendo algo. Solo viven esa experiencia y ya.

De los fragmentos presentados se extrajeron lo que a nuestro entender son los significados más ilustrativos de cada categoría. Estos nos ayudaron a hacer una serie de análisis y conclusiones preliminares con las cuales se pudo conformar un ajuste en el proceso de inducción teórica sobre la perspectiva de los fondos de conocimiento e identidad, así como de la historia oral. Este ejercicio ha de entenderse como una aproximación no concluyente con el interés de comprender las experiencias del profesorado en formación. 
TABLA 1

Continuidades/discontinuidades educativas en la formación del profesorado

\begin{tabular}{|c|c|c|c|}
\hline Categoria & Datos significativos & Análisis & Conclusiones \\
\hline $\begin{array}{l}\text { Concepción } \\
\text { sobre el } \\
\text { alumnado, }\end{array}$ & $\begin{array}{l}\text { "es importante la guía de alguien } \\
\text { para que esos aprendisajes no se } \\
\text { tornen negatitos" } \\
\text { "son como esponjas que espervan el } \\
\text { conocimiento del maestro" } \\
\text { "El alumno ha de cumplir con } \\
\text { ciertas reglas y normas. El alumno } \\
\text { ha de comportarse porque algo le } \\
\text { están enseñando en la escuela" } \\
\text { "pero el alumno es persona e } \\
\text { individuo y tiene su propia } \\
\text { interpretación del contexto. El } \\
\text { alumno aprende cuando está en } \\
\text { interacción con ese contexto y con } \\
\text { quien lo rodea" }\end{array}$ & $\begin{array}{l}\text { Los datos sugieren que el } \\
\text { alumnado aprende de todos } \\
\text { lados, pero es neoesario } \\
\text { que sea orientado para que } \\
\text { esos aprendizajes sean bien } \\
\text { utilizados. }\end{array}$ & $\begin{array}{l}\text { El profesorado en formación es } \\
\text { ambiguo, pues conciben a sus } \\
\text { estudiantes como personas que se } \\
\text { adaptan "convenientemente". } \\
\text { El alumnado es un ser activo, } \\
\text { pero en la medida en que sus } \\
\text { circunstancias lo requieran. } \\
\text { Se puede concluir que el } \\
\text { alumnado es considerado como } \\
\text { un actor pasivo sobre su propio } \\
\text { aprendizaje. }\end{array}$ \\
\hline $\begin{array}{l}\text { Relación } \\
\text { profesorado- } \\
\text { alumnado. }\end{array}$ & 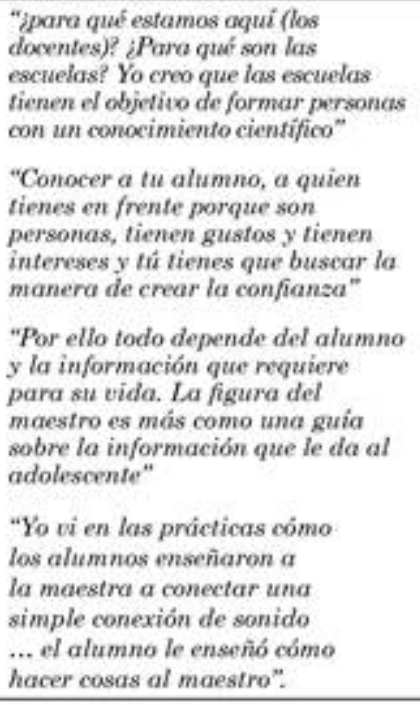 & $\begin{array}{l}\text { Según la perspectiva los fondos } \\
\text { de conocimiento e identidad } \\
\text { es fundamental conocer la } \\
\text { identidad del alumnado, así } \\
\text { como los fondos de conocimiento } \\
\text { que hereda este de su familia y } \\
\text { comunidad. En los fragmentos } \\
\text { seleccionados no se aleanza a } \\
\text { identificar con claridad esta } \\
\text { relación. } \\
\text { No obstante, se identifica } \\
\text { cierto interés por parte del } \\
\text { profesorado en formación sobre } \\
\text { aquello que hace y necesita el } \\
\text { alumnado. No es muy clara la } \\
\text { relación de interacción entre } \\
\text { docente y estudiantes. }\end{array}$ & $\begin{array}{l}\text { Aún se mantiene una } \\
\text { consideración de la enseñanza } \\
\text { como eje fundamental en la } \\
\text { relación profesorado-alumnado. }\end{array}$ \\
\hline $\begin{array}{l}\text { Relación } \\
\text { escuela- } \\
\text { familia. }\end{array}$ & $\begin{array}{l}\text { "El problema es que se le cede } \\
\text { mucho la responsabilidad a la } \\
\text { escuela como el único lugar donde } \\
\text { tienen que aprender, pero el lugar } \\
\text { donde más conocimientos generan } \\
\text { es en casa" } \\
\text { "Si se aprende algo en la escuela } \\
\text { es importante que se retomen en } \\
\text { las familias" } \\
\text { "Pero la escuela no es nada más } \\
\text { la responsable, tiene parle de } \\
\text { responsabilidad junto a la familia } \\
\text { y la sociedad" } \\
\text { "Les preguntan los padres por las } \\
\text { notas y no por las aprendisajes, Creo } \\
\text { que ello no tiene mucho sentido" }\end{array}$ & $\begin{array}{l}\text { Aunque el vínculo de la familia } \\
\text { con la escuela es deseado, pues } \\
\text { puede proveer de una relación } \\
\text { positiva, aún no es muy elaro } \\
\text { el sentido de tal relación. } \\
\text { Pareciera que se trata más } \\
\text { de una intuición que de un } \\
\text { argumento consolidado. } \\
\text { La continuidad entre familia } \\
\text { y escuela se supedita en gran } \\
\text { medida a las acciones del } \\
\text { profesorado. }\end{array}$ & $\begin{array}{l}\text { Existe una discontinuidad } \\
\text { importante, pues el profesorado } \\
\text { en formación no reconoce, } \\
\text { del todo, que es mejor una } \\
\text { redistribución equilibrada de } \\
\text { los roles de todos los actores } \\
\text { implicados (profesorado, } \\
\text { alumnado y familia). } \\
\text { Aún falta mucho por establecer } \\
\text { entre la escuela y la familia, pues } \\
\text { no se trata solo de una relación de } \\
\text { fiscalización de calificaciones, sino } \\
\text { de una acción conjunta que incida } \\
\text { en el fortalecimiento del progreso } \\
\text { académico del alumnado. } \\
\text { Se concluye que los profesores } \\
\text { y profesoras en formación } \\
\text { mantienen una discontinuidad } \\
\text { general en su práctica docente. }\end{array}$ \\
\hline
\end{tabular}

Tabla 1

Los datos sugieren que existe una desconexión del profesorado en formación con los elementos culturales del contexto educativo.; situación que evidencia la falta de continuidad entre los diferentes contextos culturales de aprendizaje e interacción de sus estudiantes. Es posible que exista una falta de congruencia entre las actividades de planeación didáctica y las necesidades de aprendizaje del alumnado. Esta conclusión 
alude al problema que hemos venido describiendo: la falta de relación entre los contextos educativos formales e informales y, por lo tanto, la inadecuada relación de conocimientos entre los diferentes contextos socioculturales del alumnado. Esto no solo supone una falta de consistencia en el proceso de enseñanza aprendizaje, sino una discontinuidad entre las actividades que se realizan en el entorno educativo formal, particularmente lo que corresponde al proceso de formación inicial. No obstante, y a pesar de las discontinuidades detectadas, se pudo documentar la necesidad, por parte de los futuros grupos docentes, de ser competentes mediante un mayor conocimiento de los mundos de vida de sus estudiantes.

\section{Conclusiones}

Consideramos que los fondos de conocimiento e identidad son una vía posible para establecer continuidades educativas. Este efecto podría conectar los diferentes contextos que conforman la ecología del aprendizaje tanto del alumnado como del profesorado en formación; situación por la que se podría superar, o cuando menos acortar, las discontinuidades entre contextos de aprendizaje. Esta conclusión implica investigar con mayor profundidad cómo generar más y mejores herramientas didácticas para fomentar y enriquecer la interacción docente-estudiante.

Este argumento, a nuestro parecer, justifica implementar el modelo de la didáctica cultural inclusiva como una estrategia pertinente para fortalecer la formación inicial del profesorado.

- Reflexionar sobre el tipo de contextos y actividades en las que estamos interconectados a lo largo y ancho de nuestra vida.

- Entender que los contextos de aprendizaje formal son insuficientes (dada la prevalencia de viejos paradigmas de formación profesional y definición de qué, cómo y para qué aprendemos) para dotar al sujeto de estrategias para aprender.

- Identificar las dinámicas y actividades de cambio en contextos de aprendizaje y conocimiento emergentes, así como sus posibles interconexiones. No solo aprendemos en espacios presenciales y sincrónicos, sino también en contextos virtuales que cada día se expanden más y que es necesario incluir como parte de nuestra experiencia de aprendizaje.

- Reivindicar que los saberes y conocimientos generados fuera de la escuela son igual o más importantes para la vida del individuo, que los desarrollados en ella. Es necesario comprender las posibles dinámicas y procesos de continuidad educativa, entre contextos de educación formal e informal, desde un marco de inclusión e interconexión.

- Establecer nuevas vías de comprensión sobre el desarrollo de la identidad. La identidad es un constructo para abordar de manera profunda las dinámicas del aprendizaje y de cómo afrontamos los cambios producidos por las dinámicas sociales actuales.

- Establecer vías de generación de aprendizajes interconectados comprendiendo el fenómeno sobre la resistencia al cambio. Cabe subrayar que no todos los sujetos están dispuestos a cambiar sus referentes sobre cómo aprendemos, situación que genera una brecha cognitiva entre personal experto y aprendiz.

- Establecer estrategias para encauzar y acompañar los procesos de aprendizaje tanto en contextos de educación formal como informal. Lo que implica investigar y diseñar instrumentos de intermediación psicológica que puedan apoyar el desarrollo de aprendizajes complejos y situados según las necesidades de aprendizaje de las personas.

- Abordar el aprendizaje en términos de continuidades/discontinudades entre contextos de educación formal e informal. Dadas las dinámicas de la SC es más significativo generar continuidades educativas entre los diversos contextos de vida del sujeto. Mientras los contextos de educación formal no tiendan puentes con otros contextos de aprendizaje las discontinuidades en el aprendizaje representarán una 
desventaja para el desarrollo de aprendizaje complejos y estratégicos para hacer frente a los retos a lo largo y ancho de la vida.

Finalmente, los puntos a seguir en esta experiencia de investigación quedan de la siguiente manera:

a) Se continuará con el desarrollo del segundo y tercer ciclo de la investigación con base en el diseño. El primer paso es la planeación de un grupo de estudio para inducir al profesorado en formación a las nociones teóricas de los fondos de conocimiento e identidad, así como de la historia oral. El supuesto es que este procedimiento pueda apoyar el cambio en los sentidos y significados sobre el papel del alumnado, la relación familia-escuela, así como la relación entre profesorado y alumnado.

b) El segundo paso será la aplicación de la historia oral al alumnado de secundaria para identificar los fondos de conocimiento e identidad. Con esta actividad se podrán elaborar secuencias didácticas que incluyan los fondos de conocimiento e identidad detectados a través del diseño de actividades de aprendizaje que establezcan continuidades educativas en la ecología del aprendizaje de estudiantes de secundaria.

c) El último ciclo de la investigación será la aplicación de estas secuencias didácticas durante las jornadas de práctica de docentes en formación. Los resultados de aprendizaje generados podrán ser la evidencia más contundente para reconocer la efectividad de la investigación. Se espera que los resultados generen mejores aprendizajes sobre los contenidos específicos, así como el desarrollo de una identidad docente más dinámica.

Como todo trabajo de investigación será el paso del tiempo y la sistematización de las experiencias lo que vaya sacando a la luz la pertinencia y efectividad del proceso de intervención educativa. Esperamos en el mediano plazo compartir, a la comunidad educativa, los resultados obtenidos y que este esfuerzo sea referente para la renovación que requieren el personal docente en México y América Latina.

\section{REFERENCIAS}

Alcocer, M. (1998). Investigación acción participativa. En J. Galindo (Coord.), Técnicas de investigación en sociedad, cultura y comunicación (pp.433-464). México: Pearson Educación.

Anderson, T. y Shattuck, J. (2012). Design-Based Research A Decade of Progress in Education Research? [Investigación basada en el diseño: ¿Una decada de progresos en investigación educativa?]. Educational researcher, 41(1), 16-25. doi: https://doi.org/10.3102/0013189X11428813

Barab, S. y Squire, K. (2014). Design-Based Research: Putting a Stake in the Ground [Investigación basada en el diseño: Colocando una estaca en el terreno]. The Journal of the Learning Sciences, 13(1), 1-14. doi: https:// doi.org/10.1207/s15327809jls1301_1

Barron, B. (2010). Conceptualizing and Tracing Learning Pathways over Time and Setting [Conceptualizando y rastreando las vías del aprendizaje a lo largo del tiempo y el entorno]. Yearbook of the National Society for the Study of Education, 109, 113-127.

Brito, L. (2014). Sociedad del conocimiento y educación: Interconexiones y posibilidades de cambio. Artículos y Ensayos de Sociología Rural, 17, 7-21.

Bronfenbrenner, U. (1987). La ecología del desarrollo humano. Experimentos en entornos naturales y diseñados. Barcelona: Paidós.

Bustos, A. y Coll, C. (2010). Los entornos virtuales como espacios de enseñanza y aprendizaje. Una perspectiva psicoeducativa para su caracterización y análisis. Revista mexicana de investigación educativa, 15(44), 163-184. Recuperado de http://scholar.google.es/scholar?q=bustos+y+coll+2010\&btnG=\&hl=es\&as_sdt=0\%2C5

Castells, M. (1994). Nuevas perspectivas críticas en educación. Barcelona: Paidós. 
Coll, C. (2009a). Enseñar y aprender en el siglo XXI: El sentido de los aprendizajes escolares. En A. Marchesi, J. Tedesco, y C. Coll (Coords.), Calidad, equidad y reformas de la enseñanza (pp. 101-112). Madrid: OEI/ Fundación Santillana.

Coll. C. (2009, septiembre). Los enfoques curriculares basados en competencias (ECBC) y el sentido de aprendizaje escolar. En Congreso Mexicano de Investigación Educativa -COMIE-, X Congreso Nacional de Investigación Educativa, Memoria (21-25). Veracruz, México.

Coll, C. (2010). Enseñar y aprender en el mundo actual: Desafíos y encrucijadas. Pensamiento Iberoamericano, 7, 44-66. Recuperado de http://www.psyed.edu.es/grintie/proGrintie/ CC_2010_Pensamientolberoamericano.pdf

Coll, C. (2013a). El currículo escolar en el marco de la nueva ecología del aprendizaje. Aula de Innovación Educativa, 2, 31-36. Recuperado de http://www.grao.com/revistas/aula

Coll, C. (2013b). La educación formal en la nueva ecología del aprendizaje: Tendencias, retos y agenda de investigación. En J. Rodríguez (Comp.), Aprendizaje y educación en la sociedad digital. Barcelona: Universitat de Barcelona. Recuperado de http://www.ub.edu/seasd/descarregues/

Coll, C. Onrubia, J. y Mauri, T. (2008). Ayudar a aprender en contextos educativos: El ejercicio de la influencia educativa y el análisis de la enseñanza. Revista de Educación, 346, 33-70.

Collado, H. (2006). ¿Qué es la historia oral? En De Garay (Coord.), La historia con micrófono. México: Instituto Mora.

Engeström, Y. (1987). Learning by expanding. An activity-theoretical approach to developmental research [Aprendizaje por expansión. Un enfoque de la teoría de la actividad para la investigación del desarrollo]. Estados Unidos de América: Cambridge University Press.

Engeström, Y. (1999). Expansive Visibilization of Work: An Activity-Theoretical Perspective [Visibilización expansiva del trabajo: Una perspectiva de la teoría de la actividad]. Computer Supported Cooperative, 8, 63-93. doi: https://doi.org/10.1023/A:1008648532192

Esteban-Guitart, M. (2012a). Towards a multimethodological approach to identification of funds of identity, small stories and master narratives [Hacia la aproximación multimetodológica para identificar fondos de conocimiento, pequeñas historias y grandes narrativas]. Narrative Inquiry, 22, 173-180. doi https:// doi.org/10.1075/ni.22.1.12est

Esteban-Guitart, M. (2012b). La multi-metodología autobiográfica extendida (MAE). Una estrategia cualitativa para estudiar la identidad, los fondos de conocimiento y las formas de vida. Revista Electrónica de Metodología Aplicada, 17(2), 51-64.

Esteban-Guitart, M. (2014). Funds of Identity [Fondos de identidad]. En T. Teo (Ed.), The Encyclopedia of Critical Psychology (pp. 752-757). New York: Springer. doi: https://doi.org/10.1007/978-1-4614-5583-7_576

Garello, V. Rinaudo, M. y Donolo, D. (2011). Valoración de los estudios de diseño como metodología innovadora en una investigación acerca de la construcción del conocimiento en la universidad. Revista de Educación a Distancia. 5, 1-34. Recuperado de http://www.um.es/ead/reddusc/5/garello.pdf

González, N. (1995). Educational Innovation: Learning from Households [Innovación educativa: aprendiendo de los hogares]. Practicing Anthropology 17,3-6. doi: https://doi.org/10.17730/praa.17.3.a036jlq42223625p

Leontiev, A. (1984). Actividad, conciencia y personalidad. México: Cartago.

Moll, L. (2014). Vygotsky and Education [Vygotsky y la educación]. London: Routledge.

Moll, L. Amanti, C. Neff, C. y Gonzalez, N. (1992). Funds of knowledge for reaching: Using a qualitative approach to connect homes and classrooms [Fondos de conocimiento para alcanzar. Usando el enfoque cualitativo para conectar los hogares y salones]. Theory into Practice, 32(2), 132-141. doi: https:// doi.org/10.1080/00405849209543534

Organisation for Economic Cooperation and Development. (2012). Connected Minds: Technology and Todays's Learners, Educational Research and Innovation. OECD Publishing. Recuperado de http:// dx.doi.org/10.1787/978926411101 
Organización de las Naciones Unidas para la Educación, la Ciencia y la Cultura. (2005). Hacia las sociedades del conocimiento. Informe mundial de la UNESCO. París: Ediciones UNESCO. Recuperado de http://redeca.uach.mx/contexto/INTRODUCCION.\%20Hacia\%20las \%20sociedades\%20del\%20conocimiento.pdf

Radzikhoskii, L. (1997). Naturaleza social de la actividad: Definiciones, fenómenos y mecanismos. Escritos de psicología, 1, 31-38.

Tedesco, J. (2011). Los desafíos de la educación básica en el siglo XXI. Revista Iberoamericana de Educación, 55(1), 31-47. Recuperado de http://dialnet.unirioja.es/servlet/articulo?codigo $=3689938$

Vélez-Ibáñez, C. y Greenberg, J. (1992). Formation and transformation of funds of knowledge among U.S.-Mexican households [Formación y transformación de los fondos de conocimiento en los hogares de mexico-americanos]. Anthropology and Education Quarterly, 23, 313-335. doi: https://doi.org/10.1525/aeq.1992.23.4.05x1582v

\section{Notas}

[4] Los autores dedican el trabajo los siguientes docentes en formación: Martha, Blanca, Susana, Hycel, Madeleim, Denisse, Nimbe, Paola, Mayra, Norma, Daniela, Iván, Giovanni, David, Aldahir, Jordan. Ustedes son parte esencial del futuro de la educación. Gracias por su apoyo y confianza.

[5] Se denomina secuencia didáctica a la unidad mínima del proceso de enseñanza y aprendizaje al reunir: a) unas intenciones educativas; b) finalidades u objetivos delimitados; c) contenidos específicos; d) actividades de enseñanza y aprendizaje que se ponen en marcha en un determinado orden para asegurar el aprendizaje de los contenidos; e) criterios, procedimientos e instrumentos de evaluación que permiten valorar el grado en que los alumnos y alumnas han aprendido (Coll, Onrubia y Mauri, 2008).

[6] En el estudio colaboraron dos psicólogos-investigadores, conjuntamente con la maestra titular de la asignatura de observación y acercamiento a la práctica, así como 16 docentes en formación (del segundo año de la Licenciatura en Educación Secundaria con Especialidad en Historia).

[7] Todos los nombres son seudónimos para salvaguardar la identidad del profesorado en formación participante.

\section{BY-NC-ND}

